\title{
Efectos de la respuesta de la demanda en usuarios residenciales sobre la reconfiguración de un sistema de distribución considerando el contexto colombiano
}

\author{
Effects of demand response in residential users on the reconfiguration \\ of a distribution system considering the colombian context
}

\author{
Millher F. Ladino ${ }^{1} \quad$ Alexis J. Bocanegra ${ }^{1}$ \\ Geovanny A. Marulanda ${ }^{1 *} \quad$ Diana Lancheros-Cuesta ${ }^{2}$ \\ Recibido 18 de junio de 2019, aceptado 13 de julio de 2021 \\ Received: June 18, 2019 Accepted: July 13, 2021
}

\begin{abstract}
RESUMEN
Los beneficios de implementar programas de Respuesta de la Demanda (RD) en grandes consumidores han sido ampliamente documentados. No obstante, aún es necesario explorar en mayor detalle los impactos de la implementación de estos programas en usuarios residenciales, sobre los beneficios de los operadores de los sistemas de distribución. En este trabajo se determinan los efectos que tendría la Respuesta de la Demanda (RD) sobre la reconfiguración de un sistema de distribución cuando se consideran las características de consumo de los usuarios residenciales en Colombia. Para esto, se construyó la curva de consumo de un usuario residencial típico en Bogotá D.C., y se tomaron mediciones que permitieron estimar la participación del usuario en un programa de RD. Además de esto, se emplea un algoritmo basado en Optimización por Enjambre de Partículas como estrategia de solución al problema de la reconfiguración. Los resultados muestran que una reconfiguración del sistema de distribución podría aumentar de manera significativa los ingresos obtenidos por un Operador de Red. En contraste, se muestra que la reducción de pérdidas de energía obtenida de la $\mathrm{RD}$, no brindaría incentivos suficientes para que estos promuevan la participación de los usuarios residenciales en este tipo de programas.
\end{abstract}

Palabras clave: Enjambre de partículas, operación de sistemas de distribución, reconfiguración de sistemas de distribución, respuesta de la demanda.

\begin{abstract}
The benefits of implementing Demand Response (DR) programs in large consumers have been widely documented. However, it is still necessary to explore in more detail the impacts of the implementation of these programs in residential users on the benefits of the distribution system operators. In this paper, we determine the effects of Demand Response $(D R)$ on the reconfiguration of a distribution system considering the consumption characteristics of residential users in Colombia. For this purpose, the demand curve of a typical residential user was constructed for Bogotá D.C., and measurements were taken to estimate the participation of this user in a DR program. In addition, an algorithm based on Particle Swarm Optimization is used as a solution strategy for the reconfiguration problem. The results show that a distribution system reconfiguration could significantly increase the revenues obtained by a Network Operator. However, it is shown that the reduction of energy losses obtained from the DR would not provide sufficient incentives for them to promote the participation of residential users in this kind of programs.
\end{abstract}

Keywords: Particle swarm optimization, distribution system operation, distribution system reconfiguration, demand response.

1 Universidad de La Salle. Facultad de Ingeniería, programa de Ingeniería Eléctrica. Bogotá, Colombia. E-mail:mladino00@unisalle.edu.co; abocanegra74@unisalle.edu.co; gemarulanda@unisalle.edu.co

2 Universidad de La Salle. Facultad de Ingeniería, programa de Ingeniería en Automatización. Bogotá, Colombia. E-mail: dilancheros@unisalle.edu.co

* Autor de correspondencia: geovanny.marulanda@iit.comillas.edu 


\section{INTRODUCCIÓN}

El crecimiento constante de la demanda y la disminución de los recursos energéticos primarios empleados en la generación de energía eléctrica, requiere que se adopten medidas en los sistemas de potencia con el fin de obtener redes más eficientes. En el caso particular de los sistemas de distribución, un aumento en la eficiencia de la red se ha logrado típicamente por medio de la ubicación de capacitores, generadores distribuidos, y la reconfiguración de la red [1]. Sin embargo, los nuevos desarrollos tecnológicos permiten emplear otras estrategias, como la coordinación de los tiempos de carga y descarga de la energía almacenada en baterías convencionales, vehículos eléctricos, y la implementación de programas de Respuesta de la Demanda (RD) [2].

La RD, se define como el cambio en los patrones o hábitos de consumo de energía eléctrica por parte de los usuarios finales en respuesta a los cambios en el precio de la electricidad en tiempo real, o a pagos de incentivos diseñados con el fin de inducir un menor consumo cuando se presentan precios elevados, o cuando la confiabilidad del sistema se encuentra en riesgo [3]. El objetivo principal de la RD es incentivar reducciones en los picos de consumo y aplanar la curva de demanda. Como resultado de esto, se obtienen beneficios potenciales como: Alivio de congestiones de la red en horas de alto consumo, aplazamiento de inversiones en infraestructura, aumento de la eficiencia, facilidad en la integración con energías renovables, y aumento en la seguridad y confiabilidad del sistema [3].

Dados los beneficios anteriores, Colombia ha manifestado un claro interés en estimular la RD en su sistema eléctrico. Puntualmente, por medio de [4], el Gobierno Nacional adopta políticas de promoción de la RD y delega a la Comisión de Regulación de Energía y Gas (CREG) las competencias para establecer mecanismos regulatorios que la incentiven. Adicional a esto, se define el Programa de Uso Racional y Eficiente de la Energía y demás formas de energía no convencionales (PROURE), como el instrumento de promoción y financiación de iniciativas que apoyen la RD. Por otro lado, en [5] se dictan los lineamientos que deberá seguir la CREG para promover la gestión eficiente de la energía y la $\mathrm{RD}$, proponiendo tarifas horarias que permitan al usuario final obtener señales de precio. Otras medidas impulsadas en Colombia pueden consultarse en [6].

Las medidas anteriores podrían extenderse hasta los usuarios residenciales, y es difícil establecer cómo podrían afectar la operación de los sistemas de distribución. A diferencia de los grandes consumidores, los usuarios residenciales no evidencian la misma racionalidad en sus hábitos de consumo, y la demanda depende de factores como el clima, los electrodomésticos instalados y las condiciones sociales [7], lo que genera un alto grado de dificultad al momento de estimar la curva diaria de carga. En este sentido, son varios los trabajos que han intentado estimar el consumo en usuarios residenciales. Por ejemplo, en [8] se propone un modelo híbrido entre mediciones históricas y modelos térmicos de la vivienda para predecir el consumo de energía eléctrica. Para esto, se tomaron los datos de consumo en una vivienda familiar con tiempos de muestreo de 5 minutos. A diferencia de [8], en [9], se estima la demanda de energía eléctrica con una resolución horaria para una vivienda partiendo de las características térmicas y los electrodomésticos instalados. En cuanto a los electrodomésticos, se identifican y modelan tanto cargas fijas como cargas que podrían desplazarse en el tiempo, y por lo tanto, ser incluidas en un programa de RD. Por otro lado, en [10] se realiza una extensa revisión bibliográfica y se analizan los factores que influyen en el consumo de energía eléctrica de un usuario residencial. En esta última referencia se identifican tres factores principales: i) Características socioeconómicas, ii) características constructivas de la vivienda y iii) tipos de electrodomésticos.

De igual forma, otros trabajos han analizado los efectos que tienen las variaciones de la demanda en la operación de un sistema de distribución. Un caso se presenta en [11], donde se analiza el efecto de la incertidumbre en la demanda sobre la reconfiguración de la red. El procedimiento propuesto, se compara con una metodología que emplea un proceso de reconfiguración estocástico de la red, combinado con el uso de Monte Carlo para simular las variaciones en la carga. Un caso similar se muestra en [12], donde se analizan las variaciones en la reconfiguración ante distintos escenarios de RD. En este trabajo, la demanda se modela en función de la elasticidad que podría 
presentar un usuario, y se emplea un flujo de potencia óptimo para determinar las tensiones que minimizan las pérdidas del sistema. Por su parte, en [13], se propone en modelo para determinar los cambios en los costos de operación y las emisiones en un sistema de distribución. El modelo propone incluir la incertidumbre en la generación eólica, solar, y la participación de usuarios en programas de RD. Las referencias anteriores muestran que la RD puede aumentar la eficiencia de la red de distribución, mejorar los perfiles de tensión y modificar los precios horarios de la energía.

Por último, en los trabajos propuestos en [14][16], se analiza el impacto que supone incluir la $\mathrm{RD}$ en los modelos de optimización empleados en sistemas de distribución. En [14] se propone un modelo no lineal en el que el operador del sistema de distribución puede reducir las cargas de algunos nodos en función de un porcentaje de su consumo. Por su parte, en [15], se busca el consumo óptimo de los usuarios en diferentes instantes de tiempo con el objetivo de minimizar el costo de operación del sistema y maximizar la comodidad de los usuarios. Tanto [14] como [15] asumen funciones continuas para la reducción del consumo que podrían no ser aplicables a usuarios residenciales cuyas cargas son, esencialmente, discretas. A diferencia de [14] y [15], en [16] se propone incluir la RD por pasos y en fracciones como una restricción dentro del problema de optimización de los recursos de una micro red. Si bien esta propuesta se acerca más al modelado de la $\mathrm{RD}$ en usuarios residenciales, en esta referencia no se consideran los hábitos de los usuarios. Otros trabajos pueden ser consultados en [17].

Teniendo en cuenta lo anterior, el presente trabajo muestra la evaluación de los efectos que tendría la $\mathrm{RD}$ sobre la operación de un sistema de distribución considerando las características generales de consumo de los usuarios residenciales en Colombia. Las principales contribuciones de este trabajo son las siguientes: i) Se propone una curva de demanda horaria para un usuario residencial típico en Colombia a partir de las características constructivas, socioeconómicas, dotación de electrodomésticos y mediciones del consumo de una vivienda. ii) Se estima la mejor participación de un usuario residencial en un programa de $\mathrm{RD}$, a partir de la identificación de carga desplazable a lo largo del día y los hábitos de consumo, iii) Los supuestos empleados para la construcción de la curva horaria de demanda de los usuarios residenciales se basan en estudios recientes realizados para la ciudad de Bogotá D.C., con el fin de hacer un estudio más realista, iv) Bajo el contexto colombiano, se calculan los beneficios técnicos y económicos que un Operador de Red (OR) podría obtener de la RD, v) Se prueba la eficacia de un algoritmo de Optimización por Enjambre de Partículas (PSO por sus siglas inglés) para solucionar el problema de la reconfiguración de sistemas de distribución considerando RD.

Para mostrar los resultados obtenidos, este trabajo se encuentra ordenado como sigue: La sección II presenta la forma en que se construyó el perfil de demanda para los usuarios residenciales, se enuncia el problema de la reconfiguración en sistemas de distribución, y se detalla el algoritmo PSO empleado en la solución del problema. En la sección III se realiza el análisis cuantitativo y cualitativo de los casos de estudio propuestos. Finalmente, en la sección IV, se presentan las principales conclusiones.

\section{METODOLOGÍA}

Para determinar el impacto que podría tener la RD en usuarios residenciales sobre la reconfiguración de un sistema de distribución, la metodología de este trabajo se dividió en dos partes. En la primera, se construyó la curva de consumo para un usuario residencial típico en Bogotá D.C., tomando como base el número de habitantes, las características constructivas y los electrodomésticos que se emplean en una vivienda típica, y se realizaron mediciones sobre un usuario residencial para estimar su participación en un programa de RD. En la segunda parte, se propone un algoritmo PSO como estrategia de solución al problema de la reconfiguración. Comparado con otras técnicas, los algoritmos PSO típicamente requieren menos tiempo de ejecución y conllevan además a resultados de una calidad similar [18], por lo que se han elegido como técnica de solución al problema de optimización. Adicional a esto, su efectividad ha sido ampliamente reportada por distintos autores en aplicaciones específicas de reconfiguración de sistemas de distribución [19].

\section{Primera parte: Caracterización de un usuario residencial típico}

Para modelar la curva de consumo de una vivienda típica de Bogotá D.C., se emplearon los resultados 
reportados en [20, 21 y 22] relacionados con el número de habitantes, las características constructivas, hábitos de consumo y electrodomésticos comúnmente empleados por usuarios residenciales. Las siguientes secciones, presentan los resultados obtenidos más relevantes y que fueron empleados para caracterizar el consumo de los usuarios residenciales en este trabajo.

\section{Número de habitantes de una vivienda típica}

De acuerdo con [22] el 40,3\% de los hogares de la ciudad Bogotá D.C. en 2014 estuvieron compuestos por cuatro o más miembros, monoparentales o biparentales, de los cuales cerca del $60 \%$ hacían parte del estrato 3 , y más del $65 \%$ habitaban en apartamentos. De manera análoga, se encuentra que un $43,6 \%$ de las personas encuestadas reportan al momento de la entrevista ser adultas, y estar entre los 25 y 59 años. Luego se encuentran las personas jóvenes entre los 18 y 24 años, que corresponde a un $13,6 \%$, y las niñas y niños entre los 6 y 12 años representan el 12,6\% del total de personas registradas. De acuerdo con lo expuesto, y con el ánimo de incorporar los rasgos más representativos de los hogares de Bogotá D.C., en este trabajo se analiza el consumo de una familia compuesta por 4 miembros, biparental, conformada por dos adultos, una persona joven, y un niño o niña.

Para asignar la ocupación de cada habitante del hogar y aproximarse a los hábitos de consumo, se observa que el 50,68\% de la población económicamente activa indican que su actividad principal en el último mes es trabajar, seguido por las que señalan estar estudiando (18,0\%). En consecuencia, para los miembros del hogar que componen este caso de estudio se asignó como ocupaciones principales: Un adulto trabaja, el otro permanece en casa, y las personas restantes estudian. En este sentido, se asignaron horarios convencionales para simular cada una de sus actividades en el hogar. La Tabla 1 presenta los horarios de permanencia de cada habitante en la vivienda a lo largo del día.

\section{Características constructivas}

Luego de consultar proyectos de vivienda en Bogotá D.C., se evidencia que un apartamento típico consta de un cuarto principal, dos cuartos pequeños, una sala-comedor, una cocina, un cuarto de ropa y dos baños. La distribución de luminarias se tomó de acuerdo a la arquitectura de cada espacio, para un total de diez luminarias. En cuanto a los televisores, se tuvo en cuenta la arquitectura de la casa, ubicando un televisor de 42" en la sala comedor, y en cada habitación, un televisor de 32". Los electrodomésticos de uso diario localizados en la cocina, consistían en un refrigerador, una licuadora y un horno microondas, y en el cuarto de ropas, se dispone de una lavadora con un uso menor durante la semana. Por otro lado, cada baño cuenta con ducha eléctrica y cada espacio de la vivienda cuenta con por lo menos una luminaria.

\section{Dotación de electrodomésticos}

La muestra más representativa de los electrodomésticos que emplean todos los estratos en la ciudad de Bogotá D.C. se evidencia en los usuarios residenciales estrato 3. En la Tabla 2 se presenta el resumen de los electrodomésticos de mayor uso, indicando la potencia nominal, el tiempo promedio de uso y la unidad de medida empleada para cada electrodoméstico. Cabe resaltar que, mientras el tiempo promedio de uso de algunos electrodomésticos dependen de las características constructivas de la vivienda, el tiempo promedio de otros electrodomésticos dependerá del número de personas que la habiten. Asimismo, su uso puede ser de varias horas o de algunos minutos según el electrodoméstico. En este sentido, se han empleado las unidades de horas o minutos, por hogar o por persona en la Tabla 2, para indicar el tiempo promedio de uso de los electrodomésticos.

Respecto del patrón de uso de las luminarias, se descarta el uso de luminarias incandescentes y se optó por asumir el uso masificado de luminarias

Tabla 1. Estadía de los habitantes en la vivienda.

\begin{tabular}{|l|c|c|c|c|}
\hline Habitante & Edad en años & Ocupación & Hora de salida de la vivienda & Hora de retorno a la vivienda \\
\hline Adulto 1 & $25-59$ & Permanece en casa & - & - \\
\hline Adulto 2 & $25-59$ & Trabaja & $08: 00$ a.m. & $05: 00$ p.m. \\
\hline Hijo 1 & $18-24$ & Estudia & 08:00 a.m. & $04: 00$ p.m. \\
\hline Hijo 2 & $6-12$ & Estudia & $08: 00$ a.m. & $04: 00$ p.m. \\
\hline
\end{tabular}


Tabla 2. Uso de electrodomésticos.

\begin{tabular}{|l|c|c|c|}
\hline Electrodoméstico & Potencia [W] & Tiempo Promedio & Unidad de medida para el tiempo promedio de uso diario \\
\hline Luminarias & 20 & No aplica & - \\
\hline Televisor 1 & 60 & 5,8 & horas//hogar \\
\hline Televisor 2 & 60 & 5,0 & horas//hogar \\
\hline Plancha & 1200 & 5,3 & minutos//persona \\
\hline Refrigerador & 100 & 24 & horas/hogar \\
\hline Licuadora & 440 & 5,3 & minutos//hogar \\
\hline Lavadora Ropa & 80 & 7,3 & minutos//persona \\
\hline Horno Microondas & 1000 & 13,6 & minutos//persona \\
\hline Ducha Eléctrica & 3750 & 6,7 & \\
\hline
\end{tabular}

de tipo LFC (Lámpara Fluorescente Compacta), en atención a la expedición del decreto 3450 de 2008, mediante los cuales se propende el uso racional y eficiente de la energía, y se invita a todos los usuarios del servicio de energía eléctrica a sustituir las fuentes de iluminación ineficientes por otras mejores, prohibiendo la comercialización en el territorio nacional de luminarias incandescentes [23]. Adicional a esto, se asocia un tiempo efectivo de uso de los espacios definidos en las características constructivas de la vivienda.

En este trabajo se denomina como "vivienda típica" aquella residencia que cumple con las características descritas anteriormente.

\section{Programas de respuesta de la demanda}

Un programa de RD es el conjunto de normas pactadas, entre participantes y prestadores de servicio, relacionadas con la forma en que los participantes gestionan su consumo, y la manera en que las empresas prestadoras de servicio se comprometen a remunerar dicha gestión. Para incentivar la participación del consumidor en programas de RD, se realizan tres acciones generales: i) los usuarios pueden reducir su demanda durante los periodos pico, esto es, cuando los precios son elevados sin modificar su patrón de consumo en las demás horas, ii) los usuarios pueden responder a los altos precios, trasladando algunas de sus operaciones de horas pico a horas no pico, y iii) los usuarios podrían optar por generar su propia energía por medio de la auto generación [24].

Por otro lado, los programas de RD se clasifican generalmente en dos categorías: Programas Basados en Incentivos (PBI) y Programas Basados en Precio
(PBP). Los PBI pueden ser divididos en programas clásicos y programas basados en el mercado. En los PBI clásicos, los clientes que participan reciben algún tipo de pago, como un crédito en la factura o un descuento por su participación en el programa. En estos programas los participantes son recompensados económicamente según la reducción de carga que alcancen durante condiciones críticas. Por otro lado, con los PBP se logra la reducción de los picos de demanda ofreciendo tarifas dinámicas a lo largo del día a los usuarios, esto es, precios altos en horas pico y precios bajos en horarios fuera del pico. En [25], se presentan las distintas alternativas de participación en programas de RD.

\section{Participación de un usuario residencial en un programa de RD}

Además de realizar la caracterización del usuario descrita previamente, se diseñó e implementó un sistema de monitorización del consumo de energía eléctrica en una vivienda que permite almacenar y consultar datos de consumo en la vivienda en tiempo real a través de un dispositivo móvil. Por otro lado, para la construcción de la curva diaria de consumo, con y sin RD, se realizó lo siguiente: i) se implementó el sistema de monitorización en la vivienda por un mes y se identificaron hábitos de consumo, ii) se observó la flexibilidad en el uso de cada electrodoméstico en la vivienda y los horarios en que podrían realizarse los máximos traslados de consumo, iii) se cruzó la información obtenida de mediciones reales con la obtenida para una vivienda típica, y se construyeron dos perfiles de demanda: Uno sin RD y otro con RD. Un resumen de la forma en que se construyó la curva de carga con y sin RD en cada nodo se muestra en la Figura 1. Con la curva de carga y el número de usuarios residenciales de cada 




Figura 1. Construcción de la curva de consumo en cada nodo con y sin RD.

nodo, se obtuvieron los consumos horarios que fueron empleados en el problema de la reconfiguración del sistema de distribución. En la sección de resultados se presentan los perfiles obtenidos. Los detalles del sistema de monitorización e información adicional de la construcción de las curvas de demanda pueden ser consultados en [26].

\section{Segunda parte: Reconfiguración del sistema de distribución}

La reconfiguración de un sistema de distribución, consiste en determinar la topología radial óptima de una red a través de la apertura o cierre de los interruptores del sistema, de forma tal, que se alcance un objetivo específico. Generalmente, el objetivo es minimizar las pérdidas para unas condiciones de carga específicas por medio de la modificación de la topología del sistema de distribución, mientras se cumplen restricciones eléctricas y operativas del sistema. En este trabajo para determinar una configuración para el sistema, esto es, los interruptores que deben permanecer abiertos y cerrados en la red, se emplea un algoritmo PSO [27, 28]. Una vez obtenida la configuración, se verifica su radialidad, se calculan sus pérdidas a través de la solución de las ecuaciones de flujo de potencia convencional, y se verifica que el sistema opere dentro de los límites técnicos.

\section{Optimización por Enjambre de Partículas}

La Optimización por Enjambre de Partículas (PSO) es una técnica inspirada en el comportamiento social de un grupo de aves (partículas) que se desplazan en un espacio de búsqueda, donde cada ave o partícula representa un punto del espacio. Las partículas (o enjambre), se mueven a través del espacio de búsqueda ajustando sus posiciones y sus velocidades según su propia experiencia y la de sus vecinos, donde cada partícula se caracteriza por su posición, velocidad y aptitud. En este trabajo, la posición de la partícula representa una configuración para el sistema de distribución, esto es, corresponde a un vector de ceros y unos que representan el estado de cada interruptor, donde el cero se ha empleado para indicar un interruptor abierto y el uno un interruptor cerrado. La velocidad, hace referencia a la probabilidad de que un interruptor cambie su estado de cero a uno en el proceso iterativo o viceversa, y la aptitud, corresponde a las pérdidas de energía obtenidas para la posición de la partícula $[18,27]$.

Para empezar con el algoritmo PSO, se generan las posiciones y velocidades iniciales del enjambre de forma aleatoria, y se seleccionan aquellas posiciones que entregan configuraciones radiales. Posteriormente, se evalúa la aptitud de cada partícula seleccionada sin incluir los límites de tensión y de corriente del sistema, seleccionando aquella que presente las menores pérdidas. Por ser la primera iteración, estos valores corresponden a la mejor posición de la partícula hasta el momento y se almacena ésta configuración como la mejor posición alcanzada por todas las partículas, esto es, la configuración que presenta la mejor aptitud en el enjambre.

Antes del cálculo de la posición de cada partícula que se empleará en la siguiente iteración, se calcula la velocidad de la partícula por medio de las expresiones ( 1 y 2) [27].

$$
\begin{gathered}
V_{i}^{k+1}=\omega V_{i}^{k}+c_{1} r_{1}\left(p_{\text {best }}^{k}-x_{i}^{k}\right)+c_{2} r_{2}\left(g_{\text {best }}^{k}-x_{i}^{k}\right) \\
\omega=\omega_{\max }\left[\frac{\omega_{\max }-\omega_{\min }}{k_{\max }} * k\right]
\end{gathered}
$$


Donde:

$V_{i}^{k}$
$c_{1}, c_{2}$

$r_{1}, r_{2}$

$k, k_{\max }$

$p_{\text {best }}^{k}, g_{\text {best }}^{k}$

$x_{i}^{k}$

$\omega, \omega_{\max }, \omega_{\min }$
Vector de velocidad de la partícula

$i$ en la iteración $k$.

Coeficientes de aceleración que relacionan la mejor posición histórica de la partícula y la mejor posición alcanzada por todas las partículas del enjambre.

Números aleatorios uniformemente distribuidos entre 0 y 1 .

Número de la iteración y número máximo de iteraciones, respectivamente.

Vectores con la mejor posición para la partícula $i$ y la mejor posición alcanzada por todas las partículas del enjambre, respectivamente. Vector de posición actual de la partícula $i$.

Factor de inercia, y valores máximo y mínimo del factor de inercia, respectivamente.

Posteriormente, se emplea la función sigmoide para actualizar la posición de cada partícula, como se presenta en (3).

$$
f\left(v_{i}^{k+1}\right)=\frac{1}{1+e^{-v_{i}^{k+1}}}
$$

Donde $f$ es la función sigmoide y $v_{i}^{k+1}$ corresponde a la posición $v$, del vector $V_{i}^{k+1}$. Finalmente, la posición de cada partícula se calcula evaluando (4) para cada interruptor.

$$
y_{i}^{k+1}=\left\{\begin{array}{cc}
1, & \text { si } r \leq f\left(v_{i}^{k+1}\right) \\
0, & \text { en otro caso }
\end{array}\right.
$$

Donde $r$ es un número aleatorio uniformemente distribuido y $y_{i}^{k+1}$ corresponde a la posición $y$, del vector $x_{i}^{k+1}$. Luego de determinar el estado de cada interruptor por medio de (4), es decir, la nueva configuración del sistema, se verifica la radialidad de la topología calculando las mallas formadas por la configuración, y calculando el número de interruptores que deberían encontrarse cerrados. Para esto, se emplean (5 y 6).

$$
N_{p}=\left(N_{i}-N_{j}\right)+1
$$

$$
N_{c}=N_{j}-1
$$

Donde:

$N_{p}$ Número de mallas de la configuración propuesta.

$N_{i} \quad$ Número total de líneas de la configuración propuesta.

$N_{j} \quad$ Número total de nodos del sistema.

$N_{c}$ Número total de interruptores cerrados.

Si la topología dada por la posición de la partícula es radial, esto es, para la configuración propuesta por el PSO se cumplen las igualdades (5 y 6), se resuelve el conjunto de ecuaciones presentado en (7 y 8). En caso contrario, se busca una nueva configuración.

$P g_{n}-P d_{n}=V_{n} \sum_{m=1}^{N} V_{m} Y_{n m} \cos \left(\theta_{n}-\theta_{m}-\varphi_{n m}\right) \quad \forall n \in N$ (7)

$Q g_{n}-Q d_{n}=V_{n} \sum_{m=1}^{N} V_{m} Y_{n m} \sin \left(\theta_{n}-\theta_{m}-\varphi_{n m}\right) \quad \forall n \in N$

Donde:

$m, n \quad$ Subíndices asociados a los nodos.

$N \quad$ Número total de nodos.

$P g_{n}, Q g_{n}$ Potencia activa y reactiva generada en el nodo $n$.

$P d_{n}, Q d_{n}$ Potencia activa y reactiva demandada en el nodo $n$.

$V_{n}, V_{m} \quad$ Tensiones en los nodos $n$ y $m$.

$\theta_{n}, \theta_{m} \quad$ Ángulos de las tensiones en los nodos $n$ y $m$.

$Y_{n m}, \varphi_{n m}$ Magnitud y ángulo de la admitancia que conecta los nodos $n$ y $m$.

Las ecuaciones (7 y 8) corresponden al balance de potencia activa y reactiva en cada nodo. La solución de estas ecuaciones permite el cálculo de las magnitudes y los ángulos de las tensiones del sistema, y el cálculo de la potencia entregada en la subestación de distribución. Una vez conocidos estos valores, es posible calcular las pérdidas de potencia activa para la configuración entregada por el PSO a través de (9).

$$
\sum_{m=1}^{N}\left(P g_{m}-P d_{m}\right)=P_{l o s s}
$$

Donde $P_{\text {loss }}$ corresponde a las pérdidas de potencia activa para la configuración entregada por el PSO, 
o lo que es igual, la aptitud de la partícula. De igual forma, es posible calcular las corrientes por cada conductor y verificar que el sistema opera dentro de los límites técnicos. Para esto, se evalúan las restricciones (10 y 11).

$$
\begin{gathered}
V_{n}^{\min } \leq V_{n} \leq V_{n}^{\max } \\
\left|I_{m n}\right| \leq I_{m n}^{\max }
\end{gathered}
$$

Donde:

$V_{n}^{\min }, V_{n}^{\max } \quad$ Límite de tensión mínimo y máximo en el nodo $n$.

$I_{m n}, I_{m n}^{\max } \quad$ Corriente y corriente máxima por la línea que conecta los nodos $m$ y $n$.

Si las tensiones y corrientes se encuentran dentro de los límites establecidos en (10 y 11), se comparan las pérdidas con el mejor resultado obtenido en otras combinaciones. Si las pérdidas son las menores obtenidas hasta el momento por la partícula, se guarda la posición de los interruptores y se almacenan como la mejor posición para la partícula, esto es, se actualiza el valor de $p_{\text {best }_{i}}^{k}$. Si las pérdidas son las menores obtenidas para todas las partículas, se actualiza el valor de $g_{\text {best }}^{k}$. En caso contrario, se procede a buscar una configuración distinta. El proceso termina cuando se alcanza un número dado de iteraciones. En la Figura 2 se presenta un diagrama de flujo con el algoritmo PSO que se ha descrito previamente.

\section{RESULTADOS Y DISCUSIÓN}

Para estimar los efectos que la RD en usuarios residenciales podría ocasionar sobre la reconfiguración de un sistema de distribución, se emplea el sistema de prueba IEEE de 33 nodos que se presenta en la Figura 3. Este sistema presenta 37 líneas, de las cuales, 32 cuentan con interruptores normalmente cerrados y 5 con interruptores normalmente abiertos. El estado de los interruptores se ha indicado en línea continua para los interruptores cerrados y punteada para los abiertos respectivamente. La tensión de la subestación ubicada en el nodo 1 es de 12,66 kV, siendo la carga total del sistema cercana a los $4.000 \mathrm{kVA}$ en la hora de máxima demanda. En la configuración original del sistema de prueba, los interruptores 33 (línea 21-8), 34 (línea 09-15), 35 (línea 12-22), 36 (línea 18-33) y 37 (línea 25-29)

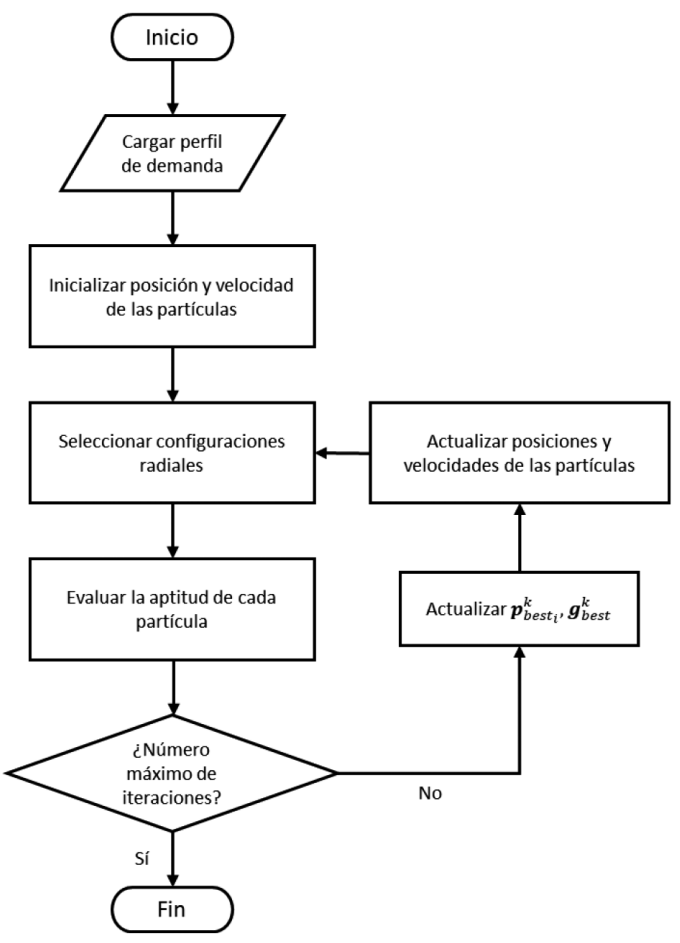

Figura 2. Diagrama de flujo PSO.

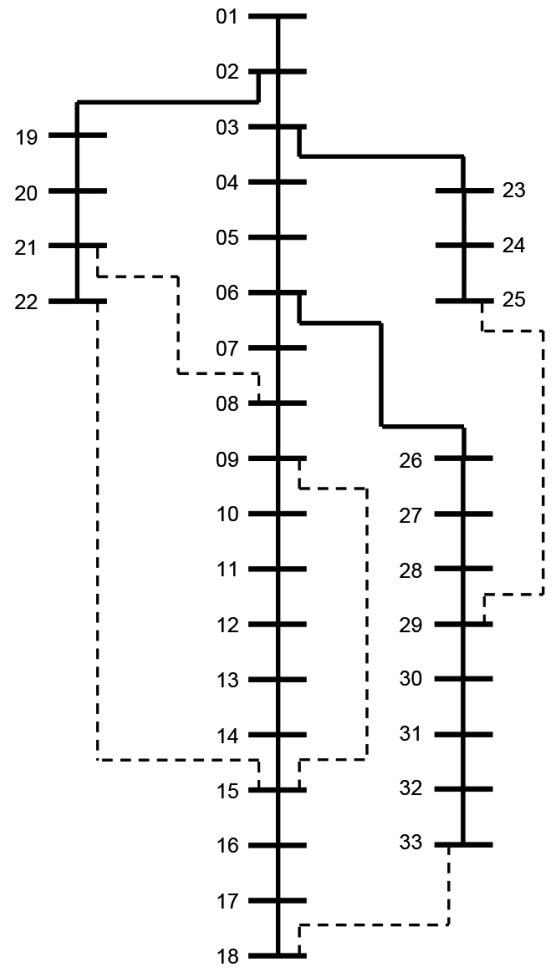

Figura 3. Sistema de prueba IEEE de 33 nodos. 
permanecen abiertos a lo largo del día. Por otro lado, para determinar las tensiones en los nodos, se ha empleado el flujo de carga radial propuesto en [29]. El número de usuarios residenciales por nodo, y datos adicionales del sistema de prueba pueden ser consultados con los autores. Por último, todas las simulaciones se han realizado empleando el software Matlab.

Los resultados de este trabajo se muestran en tres secciones distintas. La sección 1 presenta la curva de demanda obtenida para los usuarios residenciales sin y con RD. En la sección 2 se describen los resultados obtenidos para el algoritmo PSO sin incluir la RD, se analizan los efectos de la reconfiguración sobre la operación del sistema, y los beneficios económicos obtenidos por el OR. En la sección 3, se incluye la RD para los usuarios residenciales en el problema de la reconfiguración y se comparan los resultados con los obtenidos en la sección 2.

\section{Sección 1: Construcción de la curva de demanda}

De acuerdo con las consideraciones empleadas en la sección Metodología, se construyeron las curvas sin y con RD que se presentan en la Figura 4. En esta figura, el eje horizontal representa las 24 horas del día y el eje vertical el consumo de energía promedio de la vivienda en cada hora. Este valor fue obtenido como el promedio de las mediciones de potencia entregadas por el sistema de monitorización para cada hora. La línea continua de la Figura 4 y marcada con un círculo, representa la curva de demanda sin
$\mathrm{RD}$, mientras que, la línea marcada con una equis, corresponde al consumo que podría obtenerse en caso tal de implementarse un programa de RD.

Es de notarse que a lo largo del día se presenta un consumo mínimo constante debido a la nevera. En la hora 7 se tiene un incremento debido al uso de la ducha eléctrica y luminarias. A mediodía se realizan actividades domésticas, incluyendo lavado de ropa y uso de la televisión. A partir de la hora 16 se evidencia un aumento debido a la presencia del adulto 1, y los hijos 1 y 2 en el hogar. En la hora 19 se refleja el aumento del consumo dado que en esta hora no hay presencia de iluminación natural, se encuentran todos los miembros del hogar en la residencia, y se presenta el uso de varios electrodomésticos como por ejemplo luminarias, licuadora, televisores, horno microondas y plancha. Después de la hora 23 se disminuye el consumo, ya que se reduce el uso de electrodomésticos a excepción de la nevera. Finalmente, en la curva con RD se aprecian los traslados de carga de las horas 17 a 21 hacia el periodo comprendido entre las horas 12 y 16. Estos traslados se encuentran asociados principalmente al traslado del consumo de la plancha y la licuadora.

\section{Sección 2: Efecto de la reconfiguración sobre la operación}

Luego de realizar pruebas exhaustivas sobre los parámetros del PSO, los mejores resultados se obtuvieron para los siguientes valores de entrada:

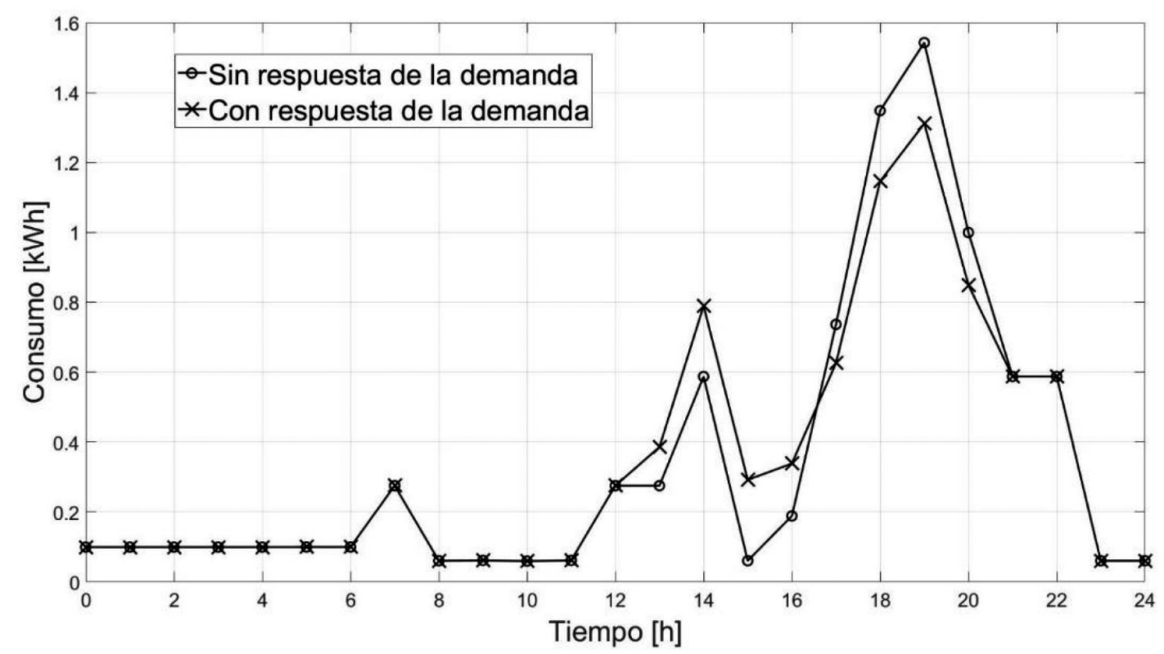

Figura 4. Curva característica de consumo típica para un usuario residencial. 
Número de partículas del enjambre $=20, k \max =60$, $\omega \min =0,4, \omega \max =0,9$ y $C 1=C 2=2,0 . \mathrm{La}$ Figura 5 presenta las características de convergencia del PSO para la reconfiguración del sistema en la hora 19. En esta figura el eje horizontal presenta el número de la iteración y el eje vertical las pérdidas de energía, de donde se evidencia una convergencia del PSO cerca de la iteración 12.

La Tabla 3, muestra los interruptores que permanecen abiertos y las pérdidas del sistema, cuando se realiza la reconfiguración del sistema a través del algoritmo propuesto. Los resultados se comparan con los obtenidos sin reconfiguración, y se evidencia una disminución de las pérdidas en cada hora, y permite alcanzar reducciones de energía cercanas a $800 \mathrm{kWh}$ diarios, es decir, reducciones cercanas al 30\% de las pérdidas de energía totales diarias.

Por otro lado, la Figura 6 presenta el efecto de la reconfiguración sobre las tensiones en todos los nodos del sistema de prueba en la hora 19 (hora pico) y sin RD. En el eje vertical se presenta la tensión en por unidad de cada nodo, donde cada nodo se ha indicado en el eje horizontal. De esta figura se puede notar que la reconfiguración del sistema de distribución mejora los perfiles de tensión en la mayoría de los nodos. Este efecto es más evidente en el nodo 18 donde la tensión cae hasta un valor de 0,91 en por unidad sin reconfiguración, mientras que con reconfiguración presenta una tensión cercana a 0,95 .
Figura 6. Tensión en los nodos sin RD para la hora 19. Además de las pérdidas y las tensiones, la reconfiguración también presenta efectos sobre los beneficios que podría obtener un OR. En Colombia, los usuarios residenciales son regulados, y el pago que reciben los comercializadores por la prestación del servicio a usuarios residenciales es establecido en la fórmula tarifaria general, y varía según el comercializador. El costo por kilovatio-hora de un importante comercializador en Colombia es de 0,13 USD aproximadamente (datos al 01 de diciembre de 2017). De manera análoga, la compra de energía por parte de los comercializadores se realiza a precio de bolsa en el mercado mayorista. Este precio varía de forma horaria y es publicado por XM [30]. Para el día tomado como referencia, el precio en bolsa por kilovatio hora osciló entre 0,06 USD y 0,09 USD. Bajo estas condiciones, el OR podría presentar un incremento en los beneficios de más de 63,000 USD diarios, debido a la disminución de pérdidas obtenidas con la reconfiguración.

\section{Sección 3: Efecto de la RD sobre la operación}

La Tabla 4, muestra las configuraciones en las horas para las cuales se presentaron cambios en la topología debido a la inclusión de la RD en el problema de la reconfiguración. De igual forma, se han resaltado los interruptores que diferencian las configuraciones. En este caso, a través de la RD se logra una disminución de las pérdidas mayor a

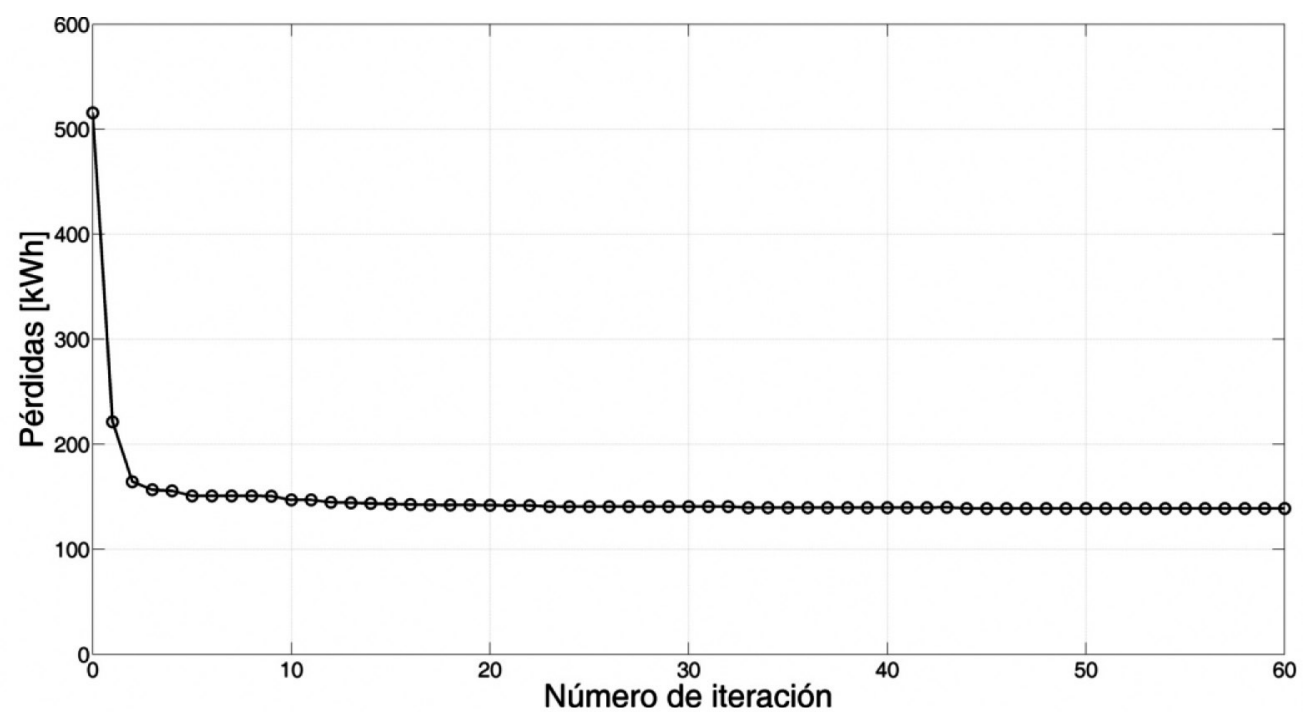

Figura 5. Características de convergencia del PSO para la hora 19. 
Tabla 3. Variaciones en la topología de la red y las pérdidas del sistema de prueba debido a la reconfiguración.

\begin{tabular}{|c|c|c|c|c|c|c|c|}
\hline Hora & \multicolumn{5}{|c|}{$\begin{array}{c}\text { Interruptores abiertos } \\
\text { con reconfiguración }\end{array}$} & $\begin{array}{c}\text { Pérdidas con } \\
\text { reconfiguración [W] }\end{array}$ & $\begin{array}{c}\text { Pérdidas sin } \\
\text { reconfiguración [W] }\end{array}$ \\
\hline 1 & 7 & 11 & 28 & 32 & 34 & 56818 & 80455 \\
\hline 2 & 7 & 10 & 14 & 28 & 30 & 48060 & 69438 \\
\hline 3 & 6 & 9 & 14 & 31 & 37 & 46630 & 66985 \\
\hline 4 & 7 & 9 & 14 & 27 & 31 & 44684 & 64964 \\
\hline 5 & 7 & 10 & 14 & 28 & 30 & 43363 & 63369 \\
\hline 6 & 7 & 11 & 14 & 28 & 31 & 43544 & 63728 \\
\hline 7 & 7 & 10 & 14 & 28 & 30 & 45319 & 65920 \\
\hline 8 & 7 & 10 & 14 & 31 & 37 & 63700 & 92182 \\
\hline 9 & 7 & 14 & 28 & 32 & 35 & 81406 & 115281 \\
\hline 10 & 7 & 9 & 14 & 28 & 32 & 85119 & 124496 \\
\hline 11 & 7 & 9 & 14 & 28 & 32 & 83627 & 118875 \\
\hline 12 & 7 & 9 & 14 & 28 & 32 & 80207 & 117063 \\
\hline 13 & 7 & 9 & 14 & 28 & 32 & 85119 & 124496 \\
\hline 14 & 6 & 9 & 14 & 32 & 37 & 76001 & 108461 \\
\hline 15 & 7 & 11 & 14 & 28 & 32 & 74037 & 106832 \\
\hline 16 & 7 & 11 & 14 & 28 & 32 & 70886 & 102125 \\
\hline 17 & 7 & 11 & 14 & 28 & 32 & 740371 & 106832 \\
\hline 18 & 6 & 9 & 14 & 32 & 37 & 116759 & 169262 \\
\hline 19 & 7 & 9 & 14 & 32 & 37 & 138928 & 208459 \\
\hline 20 & 7 & 9 & 14 & 32 & 37 & 104747 & 154476 \\
\hline 21 & 7 & 13 & 28 & 32 & 35 & 100353 & 136582 \\
\hline 22 & 7 & 9 & 14 & 28 & 32 & 84661 & 122591 \\
\hline 23 & 7 & 11 & 14 & 28 & 32 & 74037 & 106832 \\
\hline 24 & 7 & 10 & 14 & 31 & 37 & 63700 & 92182 \\
\hline
\end{tabular}

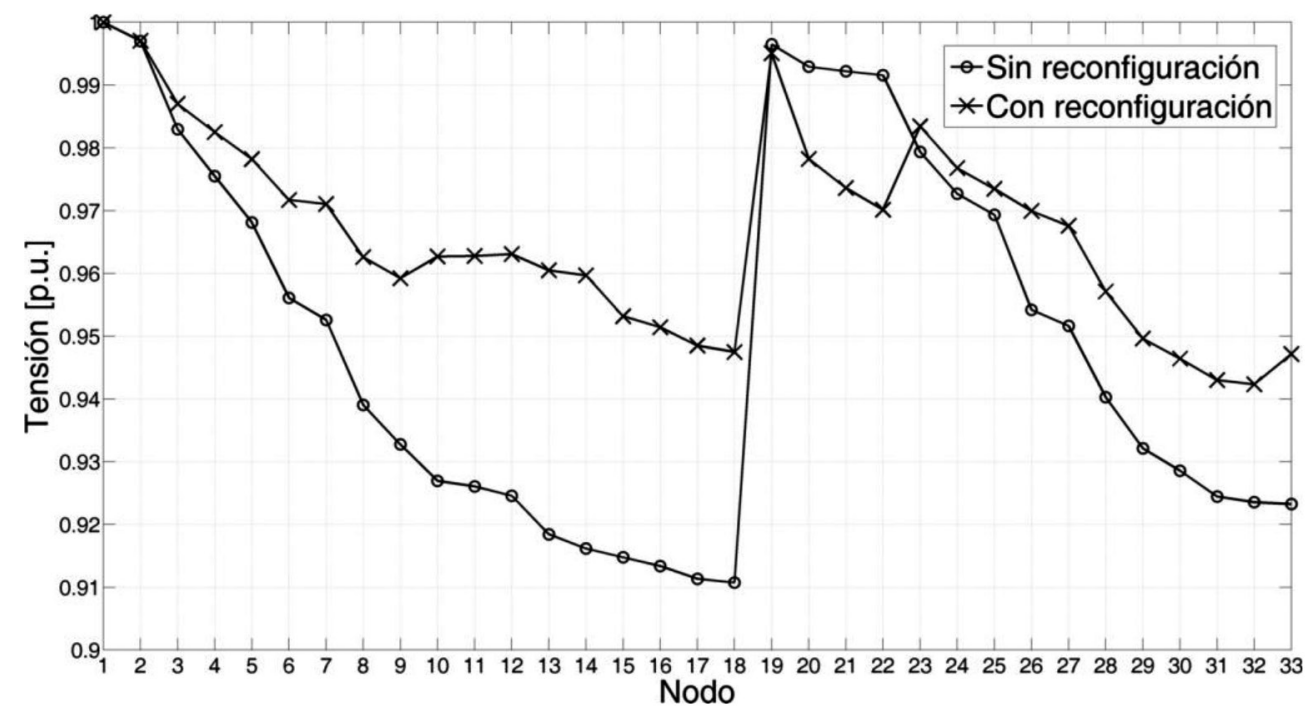

Figura 6. Tensión en los nodos sin RD para la hora 19. 
Tabla 4. Variaciones en la reconfiguración debidas a la RD.

\begin{tabular}{|c|c|c|c|c|c|c|c|c|c|c|}
\hline Hora & \multicolumn{3}{|c|}{$\begin{array}{c}\text { Interruptores abiertos con } \\
\text { reconfiguración } \\
\text { y sin RD }\end{array}$} & \multicolumn{5}{c|}{$\begin{array}{c}\text { Interruptores abiertos con } \\
\text { reconfiguración } \\
\text { y con RD }\end{array}$} \\
\hline 14 & 6 & 9 & 14 & 37 & 32 & 7 & 9 & 14 & 28 & 32 \\
\hline 15 & 7 & 11 & 14 & 28 & 32 & 7 & 9 & 14 & 28 & 32 \\
\hline 16 & 7 & 11 & 14 & 28 & 32 & 7 & 9 & 14 & 28 & 32 \\
\hline 17 & 7 & 11 & 14 & 28 & 32 & 7 & 9 & 14 & 28 & 32 \\
\hline 18 & 6 & 9 & 14 & 37 & 32 & 7 & 9 & 14 & 37 & 32 \\
\hline 20 & 7 & 9 & 14 & 37 & 32 & 7 & 10 & 14 & 28 & 32 \\
\hline 21 & 7 & 13 & 35 & 28 & 32 & 7 & 9 & 14 & 28 & 32 \\
\hline
\end{tabular}

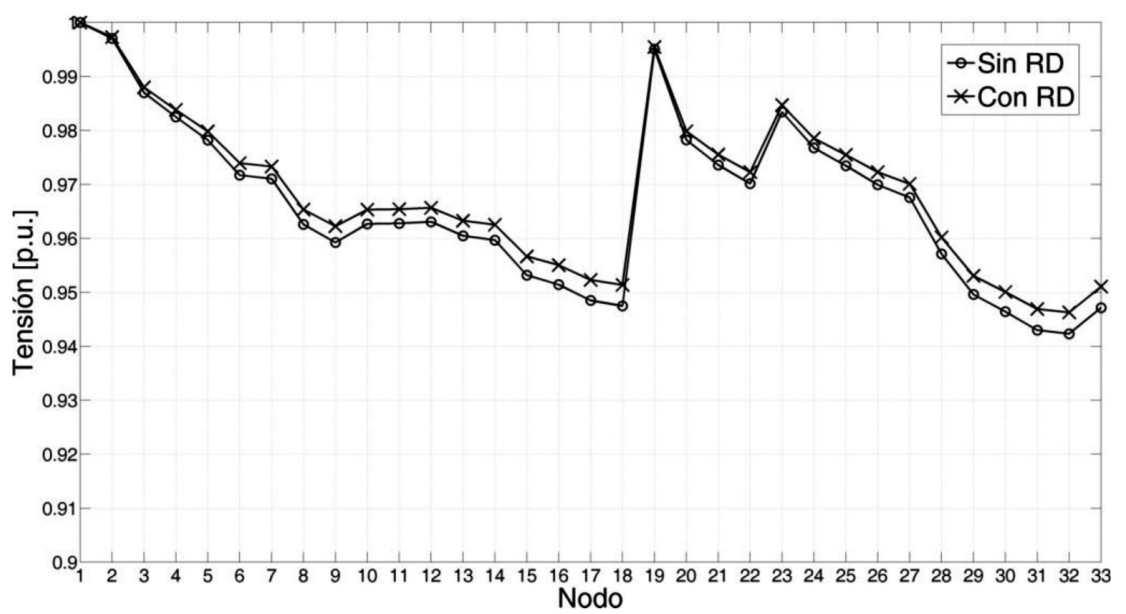

Figura 7. Tensión en los nodos con RD para la hora 19.

$14 \mathrm{kWh}$ diarias, o lo que es igual, reducciones en las pérdidas cercanas al $0,8 \%$. En términos económicos, los beneficios del OR podrían incrementarse en cerca de 1.500 USD diarios.

La Figura 7 presenta el efecto de la RD sobre los perfiles de tensión de los nodos del sistema en la hora 19. En este caso, la disminución del consumo en la hora pico, ocasiona una mejora en los perfiles de tensión de todos los nodos.

\section{CONCLUSIONES}

De acuerdo a los resultados obtenidos en este trabajo y de su análisis, se desprenden las siguientes conclusiones principales: i) Una reconfiguración horaria de sistemas de distribución podría aumentar de manera significativa los ingresos obtenidos por un OR y/o comercializador, ii) Es posible mejorar considerablemente los perfiles de tensión en los nodos a través de la reconfiguración del sistema. Estas mejoras son mayores en horas pico, iii) $\mathrm{La}$ configuración de mínimas pérdidas en un sistema de distribución cambia cuando se incluyen programas de RD. Sin embargo, los ingresos, perfiles de tensión y las pérdidas del sistema, mejoran de forma poco significativa, iv) La conclusión anterior, indica que los ingresos adicionales alcanzados por un OR a través de la reducción de pérdidas obtenidas de la $\mathrm{RD}$, no son suficientemente atractivas para que ellos promuevan la participación de los usuarios residenciales en programas de RD y v) El algoritmo PSO muestra una rápida convergencia con un bajo número de iteraciones, demostrando su eficacia en la solución de problemas de reconfiguración y RD una vez ha sido parametrizado. Finalmente, cómo trabajos futuros, se proponen: i) caracterizar la curva de demanda de usuarios comerciales e industriales en redes de distribución sin y con RD en Colombia y ii) estudiar los incentivos que tendrían los OR y/o 
comercializadores para promover la implementación de programas de RD en este tipo de usuarios.

\section{REFERENCIAS}

[1] S. Kalambe and G. Agnihotri. "Loss minimization techniques used in distribution network: Bibliographical survey". Renewable and Sustainable Energy Reviews. Vol. 29, pp. 184-200. 2014.

[2] S. Deilami and A.S. Masoum. "Real-time coordination of plug-in electric vehicle charging in smart grids to minimize power losses and improve voltage profile". IEEE Transactions on Smart Grid. Vol. $2 \mathrm{~N}^{\mathrm{o}} 3$, pp. 456-467. 2011.

[3] J.J.Cortina, J.M. López-Lezama y N. Muñoz-Galeano. "Modelo de interdicción de sistemas de potencia considerando el efecto de la respuesta a la demanda". Información Tecnológica.Vol. $28 \mathrm{~N}^{\mathrm{o}} 3$, pp. 197-208. 2017. DOI: $10.4067 /$ S0718-0 7642017000300020 .

[4] Congreso de Colombia. "Ley $1715 \mathrm{del}$ 13 de mayo de 2014". Fecha de consulta: 25 de enero 2018. URL: http://www. upme.gov.co/Normatividad/Nacional/2014/ LEY_1715_2014.pdf.

[5] Ministerio de Minas y Energía. Decreto 2492 del 3 de diciembre de 2014. Fecha de consulta: 26 de enero de 2018. URL: https://www.minminas. gov.co/documents/ 10180/23517/36863-Decreto-249203Dic2014.pdf.

[6] J.S. Gómez, S.X. Carvajal y A. Arango. "Programas de gestión de demanda de electricidad para el sector residencial en Colombia: Enfoque sistémico". Revista Energética. № 46, pp. 73-83. 2015.

[7] O. González, A. Pavas y S. Sánchez. "Cuantificación del ahorro de energía eléctrica en clientes residenciales mediante acciones de gestión de la demanda". UIS Ingenierías. Vol. 16 No 2, pp. 217-226. 2017.

[8] B. Dong, Z. Li, S.M. Mahbobur Rahmand and R. Vega. "A hybrid approach for forecasting future residential electricity consumption". Energy and Buildings. Vol. 117, pp. 341-351. 2016.

[9] A. Anvari-Moghaddam, A. Monsef and A. Rahimi-Kian. "Optimal smart home energy management considering energy saving and a comfortable lifestyle". IEEE Transactions on Smart Grid. Vol. 6 No 1, pp. 324-332. 2015.

[10] R. Jones, A. Fuertes and K. Lomas. "The socioeconomic, dwelling and appliance related factors affecting electricity consumption in domestic buildings". Renewable and Sustainable Energy Reviews. Vol. 43, pp. 901917. 2015.

[11] H. Opazo, L. García-Santander y E. López. "Reconfiguración a mínimas pérdidas, considerando la aleatoriedad de la carga: Aplicación a sistemas reales". Ingeniare. Revista Chilena de Ingeniería. Vol. $16 \mathrm{~N}^{\circ} 1$, pp. 264-272. 2008.

[12] G. Gutiérrez-Alcaraz, J.H. Tovar-Hernández and C. Lu. "Effects of demand response programs on distribution system operation". Electrical Power and Energy System. Vol. 74, pp. 230-237. 2016.

[13] A. Zakariazadeh, S. Jadid and P. Siano. "Stochastic multi-objetive operational planning of smart distribution system considering demand response programs". Electric Power System Research. Vol. 111, pp. 156-168. 2014.

[14] S. Alireza, P. Siano and A. Keane "Optimal DR and ESS scheduling for distribution losses payments minimization under electricity price uncertainty." IEEE Transactions on Smart Grid. Vol. 7 No 1, pp. 261-272. 2015.

[15] P. Samadi, A.H. Mohsenian-Rad, R. Schober, V.W. Wong and J. Jatskevich. "Optimal real-time pricing algorithm based on utility maximization for smart grid.” In 2010 First IEEE International Conference on Smart Grid Communications, pp. 415-420. 2010.

[16] J. Garcia-Guarin, D. Alvarez, A. Bretas and S. Rivera. "Schedule Optimization in A Smart Microgrid Considering Demand Response Constraints”. Energies. Vol. 13 $\mathrm{N}^{\circ}$ 17, pp. 4567. 2020.

[17] A.R. Jordehi. "Optimisation of demand response in electric power systems, a review". Renewable and Sustainable Energy Reviews. Vol. 103, pp. 308-319. 2019.

[18] F. Shariatzadeh, N. Kumar and A.K. Srivastava. "Optimal control algorithms for reconfiguration of shipboard microgrid distribution system using intelligent techniques". IEEE Transactions on Industry Applications. Vol. $53 \mathrm{~N}^{\circ}$ 1, pp. 474-482. 2016. 
[19] S. Mishra, D. Das and S. Paul. "A comprehensive review on power distribution network reconfiguration". Energy Systems. Vol. $8 \mathrm{~N}^{\circ}$ 2, pp. 227-284. 2017.

[20] Secretaría Distrital de Planeación. "Caracterización Socioeconómica: Encuesta SISBEN III". Fecha de consulta: 26 de enero de 2018. URL: http://www.sdp. gov.co/portal/page/portal/ Portal SDP/ InformacionTomaDecisiones/Estadisticas/ Bogot \% E 1\%20Ciudad \%20de\% 20 Estad\%EDsticas/2014/Caracterizacion_ SISBEN-04032015.pdf.

[21] Universidad Nacional de Colombia. "Determinación del consumo final de energía en los sectores residencial, urbano y comercial y determinación de consumos para equipos domésticos de energía eléctrica y gas". Fecha de consulta: 26 de enero de 2018. URL: http://bdigital.upme.gov.co.

[22] Secretaría Distrital de Planeación. "Encuesta multipropósito 2014 principales resultados en Bogotá y la región". Fecha de consulta: 26 de enero de 2018. http://www.sdp. gov.co/ portal/ page/portal/PortalSDP/ Encuesta_Multiproposito_2014.

[23] Ministerio de Minas y Energía. "Decreto 3450 de 2008". Fecha de consulta: 26 de enero de 2018. URL: http://historico. presidencia.gov.co/decretoslinea/2008/ septiembre/archivo.html.
[24] M.H. Albadi and E.F. El-Saadany. "A Summary of Demand Response in Electricity Markets”. Electric Power System Research. Vol. 78, pp. 1989-1996. 2008.

[25] N.G. Paterakis, O. Erdinç and J. Catalão. "An overview of demand response: Key-elements and international experience". Renewable and Sustainable Energy Reviews. Vol. 69, pp. 871-891 2017.

[26] D.J. Lancheros-Cuesta, G.A. Marulanda, M. Bueno-Lopez and D. Perez Lara. "Monitoring platform for the consumption of electricity in a home". Journal of Web Information Systems. Vol. 13 No 3, pp. 222-240. 2017.

[27] M.R. Al-Rashidi and M.E. ElHawary. "A survey of Particle Swarm Optimization Applications in Electric Power Systems". IEEE Transactions on Evolutionary Computation. Vol. $13 \mathrm{~N}^{\mathrm{o}} 4$, pp. 913-918.2009.

[28] S. Dasong, L. Junye and Z. Xiaoyang. "Application of Multi-Agent Particle Swarm Algorithm in Distribution Networks Reconfiguration". Chinese Journal of Electronics. Vol. 25 N $^{\circ}$ 6, pp. 1179-1185. 2016.

[29] W. Kersting. "Distribution system modeling and analysis". CRC press. 2012.

[30] XM. "Indicadores". Fecha de consulta: 26 de enero de 2018. http://www.xm. com.co/ paginas/Home.aspx. 